\title{
The effects of generation on auditory implicit memory
}

\author{
Ilana T. $Z$. Dew and Neil W. Mulligan \\ University of North Carolina, Chapel Hill, North Carolina
}

\begin{abstract}
The generation manipulation produces the classic functional dissociation between explicit and implicit memory. This dissociation has been explained by the transfer-appropriate processing (TAP) framework, which emphasizes the overlap in cognitive processes operative at encoding and at retrieval. However, the vast majority of implicit memory studies have been conducted in the visual modality; in the auditory modality, the effects of generation have never been investigated. In four experiments, we examined the effects of generating from semantic and nonsemantic cues on auditory implicit tests. Generating from antonyms produced a reversed generation effect on priming in auditory perceptual identification and word stem completion, while producing the traditional positive effect on explicit recognition. Generating from definitions, as well as from rhymes, also produced a reversed generation effect on auditory priming. These results are critical for more fully characterizing auditory priming and are consistent with an extended TAP analysis.
\end{abstract}

Explicit memory refers to conscious, intentional recollection of previous experiences and is assessed with direct memory tests, such as free recall and recognition. Implicit memory, in contrast, refers to nonconscious, unintentional influences of memory and is typically measured through repetition priming - in which performance on a task is facilitated (or otherwise influenced) by previous study presentation, relative to a new or baseline condition (Mulligan, 2003; Schacter, 1987). Explicit and implicit memory have been dissociated in several ways. For example, amnesic patients perform worse than normal controls on explicit tests but usually exhibit intact levels of priming (Carlesimo, 1999; Eichenbaum \& Cohen, 2001). Similar population dissociations are found between patients with schizophrenia and normal control subjects (e.g., Danion, Meulemans, Kauffmann-Muller, \& Vermaat, 2001), as well as between older and younger adults (e.g., Light, Singh, \& Capps, 1986; see Jelicic, 1995, for a review).

In addition to population differences, numerous experimental variables dissociate explicit and implicit memory (Mulligan, 2003; Roediger \& McDermott, 1993). For example, levels of processing produces a dramatic effect on explicit memory but, typically, little effect on perceptual priming. In contrast, study modality affects perceptual priming and has little effect on most explicit tests (see Roediger \& McDermott, 1993, for a review). The classic functional dissociation between explicit and implicit memory is produced by the generation manipulation, originally demonstrated by Jacoby (1983). In this study, subjects read some words without context (a target word accompanied by a neutral stimulus - e.g., XXX-cold), read some words in a meaningful context (accompanied by an antonym-e.g., hot-cold), and generated other words from the same meaningful context (e.g., hot-???). The subjects were later tested either on recognition of the target words or on the perceptual identification (PI) task, in which subjects identify briefly presented words. Jacoby found the traditional generation effect on the explicit test: Generated items were recognized better than words read in context (which, in turn, were recognized better than words in the no-context condition). However, the effect was reversed on the PI task. Identification was highest for words in the no-context condition, intermediate for the read-context condition, and lowest for the generate condition.

This reversed generation effect has been explained within the transfer-appropriate processing (TAP) framework, which emphasizes the overlap in cognitive processes operative at encoding and at retrieval. The processing overlap in the generation paradigm has been explained specifically in terms of perceptual (or data-driven) and conceptual processing (Jacoby, 1983; Roediger, 1990). Reading (especially in the no-context condition) requires subjects to process words on the basis of their surfacelevel perceptual features. Generating words from an antonym cue minimizes perceptual processing (since the target word is not written), and the words must be encoded instead on the basis of their semantic (conceptual) properties. Because the PI task requires the subject to identify words only on the basis of limited perceptual information, performance on this task should benefit from perceptual processing similar to that required at encoding and, therefore, should display the most priming for items in the read study condition (Roediger, 1990).

I. T.Z.Dew, idew@unc.edu 
Since Jacoby (1983), there has been a substantial body of research in which the generate/read paradigm has been explored with various implicit tasks (for a review, see Roediger \& McDermott, 1993; see also Mulligan, 2002). Jacoby's results have been replicated several times with a variety of perceptual priming tasks (e.g., Blaxton, 1989; Masson \& MacLeod, 1992; Srinivas \& Roediger, 1990; Toth, Reingold, \& Jacoby, 1994). Other generation manipulations, in which items are likewise generated from semantic cues, typically produce a similar, reversed generation effect on perceptual implicit tests (and often produce a positive generation effect on conceptual implicit tests; see, e.g., Blaxton, 1989; Cangoz, 2005; Smith \& Branscrombe, 1988; Weldon, 1991). Overall, the results fit well within the predictions of TAP: When generation emphasizes conceptual processing (and minimizes perceptual analysis), generation tends to produce less priming than does a comparable read condition.

The generation manipulation is important for implicit memory research for several reasons. First, it has joined other functional dissociations in helping to delineate the differences between implicit and explicit memory. Second, the reversal of the effect on explicit and implicit tests, demonstrated by Jacoby (1983) and others, has vitiated the concern that functional dissociations are solely the result of differential task sensitivity (an important concern for single dissociations between implicit and explicit tests). Third, the generation effect has been critical in the development of the TAP account of implicit memory (see, e.g., Roediger, 1990; Roediger \& McDermott, 1993). A prerequisite for the development of this account was an objective means for classifying priming tests as perceptual or conceptual (rather than a subjective classification of tasks, raising the possibility of circular reasoning). Proponents of the TAP view have used the effects of generation as a marker variable; tasks exhibiting a reversed generation effect have been classified as perceptual, and those exhibiting a positive generation effect have been considered conceptual (Roediger \& McDermott, 1993).

Despite the replicability of Jacoby's (1983) original results, some exceptions to the pattern of reversed generation effects in perceptual priming have been reported. In particular, Masson and MacLeod (1992; MacLeod \& Masson, 2000) have reported that for some generation manipulations, such as generating words from their definitions, the generation and read conditions produced equivalent levels of perceptual priming (cf. Roediger \& McDermott, 1993; Weldon, 1991). On its face, this data pattern appears to conflict with the TAP analysis. However, Masson and MacLeod (2002) concluded that the relevant generation manipulations induce covert orthographic recoding of the target item. Such processing of the item's (unpresented) visual characteristics, they argued, supports priming at a level equal to that produced by actual visual perception (in the read condition). Masson and MacLeod's (2002) analysis is generally consistent with the TAP view in attributing the high levels of priming produced by some generation conditions to prior, imagistic processing of visual word characteristics.

The vast majority of studies on implicit memory, and all of the studies on generation and perceptual priming, have used visual priming tasks. Much less is known about priming in the auditory domain. In the present study, we examined the effects of generation in auditory priming. This is critical because perceptual priming in the visual domain exhibits great stimulus and modality specificity (Schacter, Dobbins, \& Schnyer, 2004). This specificity suggests that visual priming entails modality-specific encoding operations (Schacter et al., 2004) that may differ from those underlying auditory priming. In addition, the standard multiple-memory systems account of implicit memory (e.g., Schacter, Wagner, \& Buckner, 2000) attributes perceptual priming to the operation of perceptual representation systems (PRSs), a collection of domainspecific processing modules that represent information about the form and structure of words and objects. Critically, it has been proposed that separate PRS modules process visual and auditory word form information (Carlesimo, Turriziani, \& Paulesu, 2004; Schacter, 1994; Schacter et al., 2000; Verfaellie, Keane, \& Johnson, 2000). ${ }^{1}$ Given the modality specificity of visual priming, it is important to determine whether the effects of critical variables (such as generation) produce the same effects on priming in the auditory modality as in the visual modality.

Researchers have developed auditory priming tasks that are analogous to visual priming tasks. In auditory PI tasks, spoken words are presented within noise or are passed through a filter to create a degraded or muffled stimulus, and subjects are instructed to try to identify the word. The word stem completion (WSC) task and word fragment completion task also have analogues in the auditory modality, in which portions of a spoken word are replaced with silence and the subject is instructed to complete the stem or fragment with the first word that comes to mind.

Priming in the visual and auditory modalities is comparable in several ways. Studies with amnesic patients (e.g., Schacter, Church, \& Treadwell, 1994; Verfaellie et al., 2000) and with older adults (e.g., Pilotti \& Beyer, 2002; Sommers, 1999) have produced explicit/implicit dissociations in the auditory modality that are similar to those found in the visual modality. Auditory explicit and implicit memory have also been functionally dissociated by levels-of-processing manipulations, in which conceptual encoding enhances explicit memory but not auditory priming (e.g., Schacter \& Church, 1992). In contrast, perceptual manipulations often affect auditory priming. For example, auditory study produces greater priming than does visual study on auditory priming tasks (e.g., Loveman, van Hooff, \& Gale, 2002; Pilotti \& Beyer, 2002). In addition, Church and Schacter (1994) found that changes in the speaker's voice between study and test reduced auditory priming but had little effect on explicit memory.

Taken together, these studies demonstrate that priming in the auditory modality often behaves similarly to visual priming. However, other research has pointed to potential differences between the two domains. Green, Easton, and LaShell (2001) found different amounts of cross-modal priming for nonverbal materials (e.g., seeing or hearing a baby cry) in the visual and auditory modalities: Visual processing at study produced equal priming on visual and auditory implicit tests, but auditory process- 
ing at study produced priming only in an auditory test. A second difference has come from Schacter and Church (1992): After subjects were required to focus on pitch at study, there were no priming differences in auditory PI between a pitch match and a pitch nonmatch from study to test. This result contrasts with that in a study by Graf and Ryan (1990) on visual PI, in which study-to-test changes in surface features (e.g., type font) reduced priming after surface information has been focused on at study.

Although the studies described are informative for comparing auditory and visual priming, there has been no study in which the effects of generation on auditory implicit memory have been investigated. The present experiments provide an empirical investigation of this question by examining the effects of several generation manipulations on auditory priming. This is critical for several reasons. Because priming in both the visual and the auditory domains requires at least some modality-specific processes, it cannot be assumed that the processing operations in visual and auditory priming will completely overlap in their function in regard to other critical variables, such as generation. Moreover, the effects of generation on visual implicit tests have been used by the TAP account to classify tests as perceptual or conceptual (Roediger \& McDermott, 1993); although auditory priming tests have been described as perceptual priming tasks (e.g., Blum \& Yonelinas, 2001; Loveman et al., 2002), there has been no assessment of their "perceptual" status with this important criterion.

Finally, on the basis of prior theoretical analyses of the effects of generation on priming, it was expected that auditory priming would consistently demonstrate a reversed generation effect (whereas visual priming sometimes fails to exhibit such an effect; Masson \& MacLeod, 2002). In each of the present experiments, some of the words were heard, and others were generated from semantic or nonsemantic cues. The standard TAP analysis argues that the hearing condition will produce a greater perceptual match between study and test, thus leading to a general expectation of greater priming in the hearing than in the generate condition. As was noted earlier, some generation manipulations do not produce a reversed effect in visual priming, a result due to covert orthographic processing in the generation condition, according to Masson and MacLeod (2002). The present analysis predicts that even these generation manipulations will produce a reversed generation effect in auditory priming, because orthographic processing (either overt or covert) can be expected to produce less priming on auditory tasks than in the hearing condition.

\section{EXPERIMENT 1}

An appropriate starting point for this inquiry is to examine Jacoby's (1983) classic read-generate manipulation in the auditory domain. In Experiment 1, we re-created Jacoby's within-subjects study conditions: Subjects heard some words without context; some words were heard in a meaningful context; and the remaining words were generated from an antonym cue. Auditory (rather than visual) study presentation is critical to ensure robust levels of auditory priming in nongeneration conditions (e.g., Loveman et al.,
2002; Pilotti \& Beyer, 2002). The implicit test was auditory PI in which words were masked by white noise.

\section{Method}

Subjects. Thirty-six undergraduates participated in partial fulfillment of a course requirement in an introductory psychology course at the University of North Carolina at Chapel Hill.

Design and Materials. Sixty critical items and their antonym cues were selected from materials used by Jacoby (1983). The cues were unambiguous in meaning, and the target items varied in length from four to six letters. Thirty-six of the 60 target items were indexed as high frequency by Thorndike and Lorge (1944); the remaining 24 target items were indexed as occurring 23.2 times per million (Kučera \& Francis, 1967).

The antonym pairs were divided randomly into four sets of 15 words, which were then assigned within subjects (and counterbalanced between subjects) to the following four study conditions: hearno-context, in which the target item was presented aurally following a visual series of Xs (e.g., XXXXX-"cold"); hear-context, in which the target item was presented aurally following a visual presentation of the antonym cue (e.g., hot-"cold"); generate, in which the antonym cue was presented visually followed by a series of question marks (e.g., hot-?????); and critical new, in which neither member of the pair was presented at study. The between-subjects counterbalance produced four possible study lists, so that each set of 15 items belonged to each of the four possible conditions an equal number of times across subjects. The study list was randomly ordered, with the constraint that any given condition was not presented more than two times consecutively.

One hundred twenty-two additional high-frequency words were selected from the Kučera and Francis (1967) norms (all with frequencies above 100). Sixty of the items were extracted from this set for a pilot study, which determined 5.2:1 as the appropriate white-noise:average target decibel ratio that would yield, on average, $30 \%-40 \%$ baseline correct. This ratio was used to create each of the target/white-noise files. Of the remaining 62 items from the frequency-indexed set, 2 were used as primacy buffers at study, and 60 were used as filler items on the PI test, for a total of 120 items on the test (including the 60 critical items). All critical, filler, and practice items were recorded in the same voice (that of the female experimenter) and were volume matched using the Goldwave program (Version 5.08, 2004). All items were pilot tested for baseline clarity before being masked by white noise. List order on the test was the same for all the subjects.

Procedure. The subjects were tested individually in a quiet, enclosed computer cubicle. The subjects were told that the experiment concerned attention, problem solving, and words and their meanings. Each trial of the study phase began with the presentation of the cue word (or Xs, in the no-context condition) for $3 \mathrm{sec}$. In the hear conditions, this was followed by an aural presentation of the target word over headphones. To ensure that the word was correctly heard, the subjects repeated the word aloud. In the generate condition, the cue word was followed by a (visual) series of Xs, and the subjects generated the antonym aloud. In all the conditions, continuation to the next trial was self-paced by the subjects' pressing the Enter key after speaking the target word. The experimenter followed along to ensure adherence to the study instructions. Errors in generation were tracked, but no feedback was provided to the subjects.

Following the study task, the subjects were given two 3-min distractor tasks designed to minimize recency effects and mask the purpose of the impending implicit test. The first was a sheet of 40 arithmetic problems that the subjects were asked to solve without writing any intermediate calculations. In the second distractor task, the subjects were given a set of 80 word stems to complete, with names of cities in the United States (e.g., Bos__ for Boston).

Next, the auditory PI task was administered. The subjects listened to each item presented over headphones and were instructed to try to identify each word out loud. The experimenter recorded the subjects' 
responses without providing feedback. Continuation to the next item was self-paced by the subjects' pressing the Enter key. Following PI, a questionnaire was administered to assess the extent of awareness of item overlap between the study and the test portions of the experiment. Those who reported awareness of the connection were asked whether they had consciously attempted to think back to the study list in order to improve performance on the final task. Analyses indicate that the results of the present and subsequent experiments were consistent across those categorized as test aware and test unaware (i.e., test awareness did not participate in any significant effects).

\section{Results and Discussion}

At study, 99\% of the items from the hear-no-context and hear-context conditions were repeated correctly; $90 \%$ of the items in the generate condition were generated correctly. Mean test performance for items in each encoding condition can be found in Table 1. Memory performance on the PI task was assessed with the priming measure, defined as the proportion of old items identified, relative to the proportion of critical new items identified. Using $\alpha=.05$ for all the analyses, priming scores were submitted to a one-way ANOVA, using encoding condition as a within-subjects factor. There was a significant main effect of encoding condition $\left[F(2,70)=4.12, M S_{\mathrm{e}}=.017\right]$, indicating that priming scores differed among encoding conditions. Planned comparisons revealed that priming in both the hear-no-context and the hear-context condition was greater than priming in the generate condition $[t(35)=2.46$ and $t(35)=2.68$, respectively $]$; these results indicate a reversed generation effect on auditory PI. A similar difference was found when scores from the hear conditions were compared with scores for generated items conditionalized on whether the target item was generated correctly at study $[t(35)=2.38]$. PI of items from the hear-no-context and hear-context conditions did not differ from each other $(|t|<1)$. In addition, priming was significantly greater than zero in the hear-no-context condition $[t(35)=2.748]$ and in the hear-context condition $[t(35)=2.071]$. No priming was demonstrated in the generate condition $(|t|<1)$.

The results from Experiment 1 fit well within TAP predictions. Words from the generate condition produced less priming than did words from either of the hear conditions, demonstrating a reversed generation effect. The two hear conditions did not significantly differ from each other, a result that differs slightly from that in Jacoby (1983; Roediger \& McDermott, 1993), in which priming in the read context condition was intermediate to levels of priming in the read-no-context and generate conditions. This difference will be discussed in more detail later.

\section{EXPERIMENT 1A}

The aim of Experiment $1 \mathrm{~A}$ was to demonstrate that a positive generation effect can be found with the present materials on an auditory explicit task. In the visual domain, the positive effects of generation have been widely demonstrated on explicit tests (e.g., Jacoby, 1983; Slamecka \& Graf, 1978; for a review, see Mulligan \& Lozito, 2004). In Experiment 1A, the effect of antonym generation on an auditory recognition task was examined
Table 1

Mean Proportions Correct As a Function of Encoding Condition Across Experiments 1-4

\begin{tabular}{llcccc}
\hline & & \multicolumn{3}{c}{ Encoding Condition } & \\
\cline { 3 - 4 } & Test & HNC & HC & Gen & New \\
\hline Experiment 1 & PI & .53 & .52 & .45 & .44 \\
Experiment 2 & WSC & .46 & .42 & .30 & .31 \\
Experiment 3 & WSC & - & .48 & .39 & .31 \\
Experiment 4 & WSC & - & .45 & .38 & .30 \\
\hline
\end{tabular}

Note-HNC, hear-no-context; HC, hear-context; Gen, generate; PI, perceptual identification; WSC, word stem completion.

in order to find out whether the classic functional dissociation produced by generation would be replicated in the auditory domain.

\section{Method}

Subjects. Twenty-four undergraduates participated in partial fulfillment of a course requirement in an introductory psychology course at the University of North Carolina at Chapel Hill.

Design and Materials. Study materials were identical to those used in Experiment 1. PI at test was replaced with the auditory recognition test, consisting of the 60 critical items (45 items presented at study, randomly intermixed with 15 new items). The sound files were presented in their clear, complete form during the test.

Procedure. The study and distractor phases were identical to those in Experiment 1. On the recognition test, each test item was presented over headphones. The subjects were told that some of the test items were from the study list and others were new. The subjects indicated whether the item was old or new by pressing the "o" or " $n$ " key, respectively. Instructions emphasized that words previously heard or previously generated should be considered old. The subjects were told to make their best guess if uncertain and were not allowed to skip any items. Each subsequent test item was presented $500 \mathrm{msec}$ after the prior response. Responses were tracked by the computer, and no feedback was provided to the subjects.

\section{Results and Discussion}

At study, $99 \%$ of the items from the hear-no-context condition and $100 \%$ of the items from the hear-context condition were repeated correctly; $93 \%$ of the items in the generate condition were generated correctly. Hit and false alarm rates for each encoding condition are presented in Table 2. Accuracy on the recognition test was assessed with the $d^{\prime}$ measure. Performance was best for items from the generate condition, intermediate for the hear-context condition, and lowest for the hear-no-context condition. The $d^{\prime}$ accuracy scores were submitted to a one-way ANOVA, revealing a significant main effect of encoding condition $\left[F(2,46)=14.650, M S_{\mathrm{e}}=.249\right]$. Planned comparisons indicated a significant generation effect, with the generate condition significantly higher than the hear-context condition $[t(23)=2.78]$, which in turn was significantly

Table 2

Recognition Performance Across Conditions in Experiment 1A

\begin{tabular}{|c|c|c|c|c|}
\hline \multirow[b]{2}{*}{ Measure } & \multicolumn{3}{|c|}{ Encoding Condition } & \multirow{2}{*}{$\begin{array}{c}\text { New } \\
\text { (FA Rate) }\end{array}$} \\
\hline & $\mathrm{HNC}$ & $\mathrm{HC}$ & Gen & \\
\hline Proportion "old" & .55 & .70 & .80 & .16 \\
\hline$d^{\prime}$ & 1.32 & 1.72 & 2.10 & \\
\hline
\end{tabular}

Note-HNC, hear-no-context; HC, hear-context; Gen, generate; FA, false alarm. 
higher than the hear-no-context condition $[t(23)=2.43]$. A similar difference was found when $d^{\prime}$ scores for the hear-context and hear-no-context conditions were compared with those for generated items conditionalized on whether the target item was generated correctly at study $[t(23)=3.58$ and $t(23)=5.67$, respectively $]$.

The present results indicate that the traditional generation effect can be found with the present materials for auditory recognition. Furthermore, the combined pattern of Experiments 1 and 1A closely replicates and extends the findings of Jacoby (1983); and others (Roediger \& McDermott, 1993), indicating that the pattern of opposite effects of generation on recognition and PI extends to the auditory modality. This serves to support functional independence between these auditory explicit and implicit memory tests.

In research on implicit memory, it is always important to consider the possibility of explicit contamination on the implicit measure. In Experiment 1, we followed the design suggestions of Roediger and McDermott (1993) in order to minimize this likelihood. Per Roediger and McDermott's guidelines, Experiment 1 had the following features: (1) The study (and test) instructions were incidental; (2) the test instructions emphasized providing the first response that came to mind; (3) multiple filler tasks intervened between the study and the test portions of the experiment; (4) the proportion of old items on the implicit test was below 50\%; and (5) the implicit test began with filler items not from the study portion of the experiment. Roediger and McDermott argued that these design features would limit both the likelihood and the utility of explicit contamination. Experiment 1A provides further evidence on this point. The opposite pattern of performance on the implicit and explicit tests provides additional evidence against explicit contamination. If explicit retrieval had been used to aid performance on PI, it would have benefited the items in the generate condition. However, generation produced no priming in Experiment 1.

\section{EXPERIMENT 2}

To gain a broader understanding of priming in the auditory domain, it is important to generalize the results of Experiment 1 to other implicit tests. Experiment 2 extended the analysis of generation effects to auditory WSC. This experiment was conducted for several reasons. First, we had found that auditory WSC produced greater priming than did auditory PI (Mulligan, Duke, \& Cooper, 2007); thus, auditory WSC may allow for greater sensitivity in detecting smaller priming differences among encoding conditions. Second, differences between visual PI and WSC have occasionally been found following generation (e.g., Schwartz, 1989). Third, differences have been reported between auditory PI and WSC in other auditory-priming experiments. Most critically, the effects of study modality have uniformly been found for auditory PI (Pilotti, Bergman, Gallo, Sommers, \& Roediger, 2000; Pilotti \& Beyer, 2002; Pilotti, Meade, \& Gallo, 2003) but have been less robust for auditory WSC, with some studies showing no effect (Berry, Banbury, \& Henry, 1997; Blum \& Yonelinas,
2001), others showing a significant effect (Pilotti, Bergman, et al., 2000), and still others showing an effect of study modality only when responses were spoken but not written (Loveman et al., 2002). In addition, Pilotti, Bergman, et al. (2000) found that even when WSC was affected by study modality, it was unaffected by a study-test voice change that reduced priming in auditory PI. This pattern of results implies that auditory WSC may be less sensitive to manipulations of perceptual form than is auditory PI, which further raises the possibility that auditory WSC may be less likely to exhibit an effect of surface form manipulation as implemented in the generation task. On the other hand, the standard TAP analysis, as detailed earlier, argues that perceptual priming tasks (such as WSC) should generally exhibit the reversed generation effect.

\section{Method}

Subjects. Twenty-four undergraduates participated in partial fulfillment of a course requirement in an introductory psychology course at the University of North Carolina at Chapel Hill.

Design, Materials, and Procedure. The study phase and the distractor tasks were identical to those used in Experiment 1. PI at test was replaced by WSC. To create the test stimuli, the critical items from Experiment 1 (not masked by noise) were edited so that only the first one or two phonemes of the item could be heard (the remainder of the sound file was replaced with silence). As is typical for WSC, the stems were constructed to allow for multiple legitimate completions. Word stems were pilot tested to produce $30 \%-40 \%$ accuracy in baseline critical response. The subjects listened to each word stem presented over headphones and were instructed to fill in each stem with the first word that came to mind that could complete the stem. If they were unable to think of a completion, they were instructed to move on to the next word stem. As in Experiment 1, continuation to the next item was self-paced by pressing the Enter key.

\section{Results and Discussion}

At study, $100 \%$ of the items from the hear-no-context and hear-context conditions were heard correctly; $94 \%$ of the items in the generate condition were generated correctly. Mean test performance for items from each encoding condition is included in Table 1 . There was a significant main effect of condition $\left[F(2,46)=7.80, M S_{\mathrm{e}}=\right.$ $.021]$, indicating that priming scores differed among encoding conditions. Planned comparisons revealed that priming was greater in both the hear-no-context and the hear-context conditions than in the generate condition $[t(23)=4.27$ and $t(23)=2.56$, respectively $]$; these results indicate a reversed generation effect on auditory WSC. A similar difference was found when scores from the hear conditions were compared with those for generated items conditionalized on correct generation at study $[t(23)=$ 3.58]. The hear-no-context and hear-context conditions did not differ from each other $(|t|<1)$. Priming was significant in the hear-no-context condition $[t(23)=5.08]$ and in the hear-context condition $[t(23)=3.51]$, but not in the generate condition $(|t|<1)$.

Experiment 2 revealed a pattern of priming performance that was the same as that in Experiment 1. Performance was significantly higher in the hear conditions than in the generate condition, demonstrating a reversed generation effect. As was expected, overall priming scores were somewhat higher for WSC than for PI, allowing 
greater sensitivity in detecting small differences. Still, no significant difference was found between the two hear conditions, providing further evidence that this is a reliable effect.

Some studies have demonstrated differences between auditory PI and WSC in their sensitivity to perceptual information (e.g., Pilotti, Bergman, et al., 2000). Despite any potential differences for auditory PI and WSC in the effects of study modality, the present generation manipulation produced similar effects: Both tests exhibited a reversed generation effect and no significant priming in the generation condition. This was similar to the results in the bulk of the studies in the visual modality, which have shown that the reversed effect following semantic generation can be generalized across implicit tests (see Roediger \& McDermott, 1993). It also helped to motivate Experiment 3, which was intended to generalize the results of Experiments 1 and 2 to a different semantic generation task.

\section{EXPERIMENT 3}

In Experiment 3, we examined the effect of generating from definitions on auditory WSC. In visual priming, antonym generation has consistently produced a reversed generation effect (for a review, see Roediger \& McDermott, 1993). However, other semantic generation tasks, such as generating from a definition, have produced somewhat mixed results. As was noted earlier, some studies (e.g., Masson \& MacLeod, 1992) have reported no effect with this generation manipulation, whereas most other studies (e.g., Clarke \& Morton, 1983; Weldon, 1991; Winnick \& Daniel, 1970) have reported a reversed generation effect consistent with the results of antonym generation. Consequently, it was critical to determine whether the reversed generation effect found in Experiments 1 and 2 would generalize to other generation manipulations. Of course, the standard TAP analysis predicts a reversed generation effect. Likewise, Masson and MacLeod's (2002) account predicts a reversed generation effect. Masson and MacLeod (2002) explained the equivalent visual priming produced by reading and generation from definitions by positing covert orthographic processing in the generation condition. Such processing should not transfer well to auditory priming, and so this account also predicts a reversed generation effect in the present instance.

The study phase of the present experiment included only words heard in context and generated words, for several reasons. First, Experiments 1 and 2 indicated a negligible difference in priming for words in the hearno-context and hear-context conditions. Second, the effect of generation (which was our central interest) could be evaluated only by holding context constant across encoding conditions. Third, the HNC condition was included in the earlier experiments to facilitate comparison with the original Jacoby (1983) results; in other studies of generation in the visual modality, only read and generate conditions were used in which context was held constant, as in the present experiment.

\section{Method}

Subjects. Thirty-two undergraduates participated in partial fulfillment of a course requirement in an introductory psychology course at the University of North Carolina at Chapel Hill.

Design and Materials. Experiment 3 was the same as Experiment 2 , except for the following modifications. In the study phase, antonym pairs were replaced with items and their definitions. Simple, unambiguous definitions to 80 critical items were created and pilot tested, producing at least $88 \%$ correct generation. All the critical words were one syllable and five letters in length, with a mean frequency of 43 (range, 20-88; Kučera \& Francis, 1967). The critical items were randomly divided into two sets of 40 for the old-new counterbalance. Twenty words on each study list were presented as hear-in-context. Targets were presented aurally following a 3-sec visual presentation of the definition (e.g., move to music-"dance"). The remaining 20 were in the generate condition, in which words were generated from visual stems following a 3-sec definition cue (e.g., move to music-“da"). The additional letter clues were important to ensure appropriate levels of correct generation. In total, this produced four different study lists, which were assigned to equal numbers of subjects. The study list was randomly ordered, with the constraint that no more than 2 items of the same condition were presented consecutively.

The word stems for the test portion were created the same way as in Experiment 2. A total of 115 items was included on the test: 80 critical items (40 old and 40 new) and 35 filler items, which were similar in length and frequency to the critical items.

Procedure. The procedure was the same as that in Experiment 2, with the following modifications to the study phase. In the hear condition, the subjects were instructed to read the definition silently, listen to the presented word that corresponded to the definition, and repeat the spoken word out loud. In the generate condition, the subjects were instructed to read the definition silently, generate a word that corresponded with the definition and that began with the presented letters, and say the generated word out loud. Two practice items (one of each study instruction) preceded the critical items. As in Experiments 1 and 2, the experimenter followed along to ensure adherence to the study instructions. Errors in generation were tracked, but no feedback was given to the subjects.

\section{Results and Discussion}

At study, $100 \%$ of the items from the hear condition were heard correctly; $92 \%$ of the items in the generate condition were generated correctly. Mean test performance for the items from both encoding conditions can be found in Table 1 . There was a significant main effect of condition $[t(31)=3.08]$, indicating that priming was greater in the hear than in the generate condition; a reversed generation effect on auditory WSC was again found. A similar difference was found when scores from the hear conditions were compared with those for generated items conditionalized on correct study generation $[t(31)=2.3]$. Priming was significantly greater than zero in both the hear condition $[t(31)=6.29]$ and the generate condition $[t(31)=2.93]$.

The results of Experiment 3, like those of Experiments 1 and 2, fit with the TAP-based predictions. The generate condition produced significantly lower priming on the auditory implicit test than in the hear condition, demonstrating that a reversed generation effect can be generalized to semantic generation tasks other than antonym generation. One minor difference is that in Experiment 3, significant priming was produced for items in the generate condition. This result is secondary to the comparison of interestpriming in the hear condition, relative to the generate 
condition - and is not unprecedented in the literature; several studies have shown that generation can produce significant visual priming (e.g., Schwartz, 1989). This issue will be explored further in the General Discussion section. The key result here is the increase in priming for the heard items, relative to the generate items, demonstrating the expected reversed generation effect.

\section{EXPERIMENT 4}

Experiments 1-3 showed that the reversed generation effect occurs for two different auditory priming tasks using two different manipulations of generation. It should be noted that both generation manipulations contrasted a perceptual condition with a semantic generation condition. Experiment 4 further examined the generality of the reversed generation effect by using a nonsemantic generation manipulation, in which a critical item either was generated from a phonological (rhyme) cue or was heard in the presence of the same cue. This generation task provides an important assessment of the standard TAP analysis in two ways.

First, this manipulation raises the question of the appropriate form of the TAP account. As was described in the introduction, the TAP account is typically framed in terms of the contrast between conceptual and perceptual processes (e.g., Jacoby, 1983; Roediger \& McDermott, 1993). The standard TAP account proposes a conceptualperceptual trade-off, in which the generation condition enhances conceptual processing and, thus, transfers poorly to a perceptually driven implicit test, whereas the visual (or hearing) condition accentuates perceptual processes, transferring well to a visual (or auditory) priming task. However, the perceptual-conceptual distinction is a specific instantiation of the more general notion of TAP, which states that the overlap in encoding and retrieval processes dictates memory performance.

Importantly, not all generation manipulations emphasize conceptual processing. For example, the rhyme generation task used in the present experiment does not require conceptual or semantic analysis of the target item, even though it enhances explicit memory (Mulligan, 2002; Mulligan, Lozito, \& Rosner, 2006; Slamecka $\&$ Graf, 1978). The general TAP principle suggests that rhyme generation should nevertheless produce a reversed generation, because the hearing condition is still a better match for the later auditory priming task than is the generation task. ${ }^{2}$ Thus, a generation task that does not induce a perceptual-conceptual trade-off in encoding is still expected to produce a reversed generation effect. This implies a more generic version of the TAP account of generation effects, differentiating between a hearing condition that produces greater auditory processing of the target stimulus and a generate condition that produces less, regardless of whether the generation task is semantic or nonsemantic.

Second, the rhyme generation task assesses the specificity of the perceptual processing required to produce full priming (i.e., to the level produced by actual perception in the hear condition). The general TAP view predicts a reversed generation effect, as described above, because the hearing condition more closely matches the demands of the auditory priming task. However, the generate condition requires substantial processing of sound (specifically, phonological) information in order to generate the word, which might support high levels of auditory priming. Prior research in the auditory domain has not clarified the extent to which generating from auditory characteristics functionally resembles the perceptual operations involved in hearing. In some studies (e.g., Stuart \& Jones, 1996), internally generating an auditory stimulus by imaging a spoken version of a written word has produced levels of auditory priming equivalent to those produced by directly hearing the words, but in other studies (e.g., Pilotti, Gallo \& Roediger, 2000), it has produced less priming than has hearing. In both of these manipulations, however, the items were presented in complete visual form, which reduces the resemblance to more typical generation tasks. Therefore, it is critical to determine whether a reversed generation effect will occur for a generation manipulation based on nonsemantic, phonological analysis of rhyme cues.

\section{Method}

Subjects. Thirty-two undergraduates participated in partial fulfillment of a course requirement in an introductory psychology course at the University of North Carolina at Chapel Hill.

Design, Materials, and Procedure. Experiment 4 was the same as Experiment 3, except for the following modifications. In the study phase, definitions were replaced with familiar words that served as unambiguous rhyme cues. The critical items consisted of the 80 critical items used in Experiment 3, plus 8 additional items similar in length and frequency. The critical items were randomly divided into two sets of 44 for the old-new counterbalance. Twenty-two words on each study list were presented as hear-in-context: Targets were presented aurally following a 2-sec visual presentation of the rhyme (e.g., sound-"round"). The remaining 22 were in the generate condition, in which words were generated from visual stems of two letters following a 2-sec rhyme cue (e.g., sound-ro). The additional letter clues were important for ensuring appropriate levels of correct generation; as in Experiment 3, these clues were visual, and thus no auditory information was presented in the generate condition. Rotating the critical items through old-new and read-generate conditions produced four different study lists, which were assigned to equal numbers of subjects. The study list was randomly ordered, with the constraint that no more than 2 items of the same condition were presented consecutively. In the hear condition, the subjects were instructed to read the first word silently, listen to the presented word that rhymed with the first word, and repeat the spoken word out loud. In the generate condition, the subjects were instructed to read the first word silently, generate a word that rhymes with it and that begins with the presented letters, and say the generated word out loud. Two practice items (one of each study instruction) preceded the critical items.

The word stem completion test consisted of stems corresponding to 123 items: 88 critical items ( 44 old and 44 new) and 35 filler items, which were similar in length and frequency to the critical items.

\section{Results and Discussion}

At study, $99.6 \%$ of the items in the hear condition were heard correctly; $98.3 \%$ of the items in the generate condition were generated correctly. Mean test performance for items from both encoding conditions can be found in Table 1. There was a significant main effect of condition $[t(31)=2.5]$, indicating that priming was greater in the 
hear than in the generate condition; a reversed generation effect on auditory WSC was again found. An identical pattern was found when scores from the hear conditions were compared with those for generated items conditionalized on correct study generation $[t(31)=2.5]$. Priming was significantly greater than zero in both the hear condition $[t(31)=4.7]$ and the generate condition $[t(31)=2.6]$.

In Experiment 4, as in Experiments 1, 2, and 3, the generate condition produced significantly lower priming on the auditory implicit test than did the hear condition, demonstrating that a reversed generation effect can be generalized to tasks other than semantic generation. Not only does this finding represent an empirical generalization of the results of the earlier experiments, it also implies a theoretical generalization of the standard TAP account. This standard account describes the generation manipulation as inducing a conceptual-perceptual trade-off. However, inherent in the general TAP principle is the prediction that any generation manipulation not based on actual perception of the target item should produce the same reversed generation effect on perceptual implicit memory. The present finding accords with this more general version of the TAP account.

Although the generation manipulation required the subjects to analyze phonological features rather than conceptual features, later performance on auditory WSC was facilitated to a greater extent by direct auditory input in the hear condition. The pattern revealed by this comparison is consistent with other, similar findings in the auditory priming literature (e.g., Pilotti, Gallo, \& Roediger, 2000; but cf. Stuart \& Jones, 1996) and emphasizes the importance of direct perceptual overlap between study and test for performance on auditory priming. Merely processing phonological information was not enough to produce maximal levels of auditory priming.

\section{GENERAL DISCUSSION}

In summary, Experiment 1 compared the effects of hearing words without context, hearing words in a meaningful context, and antonym generation on auditory PI. A reversed generation effect was found, with larger amounts of priming in the hear conditions, relative to the generate condition. This reversed generation effect was generalized to auditory WSC in Experiment 2, to generation from definitions in Experiment 3, and to phonological generation in Experiment 4. In all the experiments, priming in the hear conditions was significantly greater than priming in the generate condition (which was nonsignificant in Experiments 1 and 2). Lastly, the positive generation effect found on auditory recognition in Experiment 1A demonstrated that Jacoby's (1983) classic dissociation between explicit and implicit memory could be replicated in the auditory domain; this anticipated dissociation is consistent with the described bodies of research on the effects of generation on implicit memory. The results of the recognition test also provide evidence against the possibility that explicit retrieval strategies affected performance on the implicit tests; if consciously controlled processes had been used to aid performance, they would have benefited items in the generate condition.
The results show that generation joins other manipulations reported in the literature that have demonstrated important similarities between visual and auditory priming. As was described earlier, explicit memory has been dissociated from implicit memory in both the visual and the auditory modalities. Amnesic patients and older adults often demonstrate impaired performance on explicit tasks, as compared with largely intact performance on both visual and auditory implicit tasks (e.g., Schacter et al., 1994; Sommers, 1999). Visual and auditory priming have also shown sensitivity to changes in the perceptual features of the target stimuli, a manipulation that typically has little or no effect on explicit tests such as recognition (e.g., Church $\&$ Schacter, 1994; Pilotti, Bergman, et al., 2000). The present experiments demonstrate that generation also produces a functional dissociation between explicit recognition and auditory priming. The direction of the effect of generation on later implicit tests has often been used by proponents of TAP to classify these tests as perceptually driven. The reversed generation effect on auditory PI and WSC found here supports the classification of these tests as perceptual as well. Doing so may support a more general functional overlap between visual and auditory priming.

The present results fit well within the TAP analysis. This traditional TAP account, based on the conceptualperceptual distinction, predicts that perceptual retrieval tasks will benefit from perceptually driven encoding tasks and will be largely insensitive to conceptual encoding conditions. Consistent with this prediction, auditory PI and WSC demonstrated the best performance for items that had been processed aurally at study and did not benefit from semantic generation encoding. The results of Experiment 4 extend the standard TAP analysis in implying that the generation task does not have to involve conceptual analysis. Priming was facilitated for words that were processed aurally, relative to phonological generation, which emphasizes the benefit of direct perceptual input for auditory WSC. This is consistent with the more general TAP principle underlying the conceptual-perceptual distinction, indicating that a trade-off in perceptual and conceptual processing is not required. A more generic TAP account suffices: Provided that the hear condition produces greater perceptual overlap with test, the reversed generation effect is to be expected, regardless of the semantic or nonsemantic nature of the generation manipulation.

Although the key results of the present experiments support the established similarities between visual and auditory priming, there is one interesting difference to consider. As was mentioned earlier, Jacoby (1983) and others (reviewed in Roediger \& McDermott, 1993) found that reading a word in the context of its antonym produced less priming than did reading the word without context. In our Experiments 1 and 2, hearing a word with or without context produced comparable levels of auditory priming. It should be noted that in standard visual experiments (as used by Jacoby, 1983), a target word and its contextual cue are presented at the same time, whereas the auditory presentation in the present experiments necessitated that the context word precede the (auditory) target word (simultaneous presentation in the auditory modality would 
be distracting or confusing). Jacoby demonstrated in the visual domain that a delay between the presentation of the cue and the visual target word further reduced priming. Consequently, in our experiments, presentation parameters were used that maximized the effects of context in the visual modality, yet only nonsignificant trends were found in auditory priming.

One possible explanation hinges on a methodological difference: In the present context condition, the cue word was presented visually and the target word aurally, whereas in Jacoby's (1983) context condition, both words were presented visually. It is possible that a perceptually isolated target word produces greater priming (e.g., MacLeod \& Masson, 2000). Alternatively, auditory perception of words may be more obligatory than visual perception of words and, so, less influenced by context. Subsequent research is required to determine whether the context effects found in visual priming (e.g., Levy \& Kirsner, 1989; Martin-Chang \& Levy, 2006) can be found in auditory priming. If not, the effects of context would amount to the first substantial difference in priming between the two modalities.

One other aspect of the results merits discussion. In Experiments 3 and 4, significant priming was found for items generated from definitions and rhymes, whereas in Experiments 1 and 2, antonym generation produced no significant priming. First, it should be noted that this pattern is not unprecedented. In the visual domain, both Jacoby (1983) and Masson and MacLeod (1992) reported nonsignificant priming for antonym generation, whereas Masson and MacLeod (1992), Schwartz (1989), and Weldon (1991) found significant priming in a definition generation condition (see MacLeod \& Masson, 2000, for a similar result). Second, the requirement to say the words aloud in the generate condition cannot account for the above-chance priming in Experiments 3 and 4, since the same requirement was in place for Experiments 1 and 2 (more on this momentarily). Why might this result occur? Masson and MacLeod (1992, 2002; MacLeod \& Masson, 2000) have considered reasons why some generation conditions produce substantial visual priming and others do not. As was noted earlier, Masson and MacLeod (2002) argued that some generation cues (such as definitions) induce covert orthographic processing of the target item, whereas other generation conditions (such as antonym generation) do not. In the present case, this seems unlikely to have produced the observed pattern of results. If generation from definitions and rhymes induces orthographic processing, such processing may well enhance visual priming but will be less likely to aid auditory priming.

Masson and MacLeod (1992; MacLeod \& Masson, 2000) considered another possibility, that differences may stem from the contextual boundedness of the initial encoding of the target item. According to this view, antonym pairs are so strongly associated (or so well integrated) that generating a word from its antonym produces a contextually bound interpretation of the target word, an interpretative encoding that does not transfer well to the test in which the item is presented in isolation. Reading a word in the same context produces less contextual boundedness, because the target item can be identified on the basis of the presented visual features, rather than from the meaning of the antonym cue. Masson and MacLeod (1992) argued that definitions (and presumably, rhymes) are not as strongly associated to (or integrated with) their target items and so do not produce the same type of contextually bounded encoding. This produces (at least some, above-chance) priming on the subsequent single-item test. This account is speculative, of course, but seems to fit the difference in priming found across difference types of generation tasks. Critically, despite such distinctions, priming of generated items in all the experiments reported here was less than that produced by directly hearing the target items, consistent with the more general pattern predicted by TAP.

In Experiments 1 and 2, no priming occurred in the generate condition, a result consistent with typical antonym generation in the visual modality. This result merits consideration, however, given that the subjects generated the critical items out loud. This result interests us because it cannot be fully explained by the mismatch in the perceptual stimulus between the test stimulus (recorded in the experimenter's voice) and the subject's own voice. The perceptual mismatch, of course, was further magnified because the subjects wore headphones, thereby muffling the sound of their voices. In several prior studies, however, some auditory priming occurred when the surface-level features of the stimulus did not match from study to test. Church and Schacter (1994) demonstrated some priming when the fundamental frequency of the auditory stimulus changed. Furthermore, Pilotti, Gallo, and Roediger (2000) found reduced but still significant priming when subjects imagined the word being spoken but did not actually hear it. The result in the present study, therefore, has possible implications for the auditory processing of self-produced stimuli. If the perceptual mismatch between study and test does not fully explain the lack of priming, other factors could be considered, such as the extent to which subjects perceptually process or attend to self-produced stimuli. Regardless, generation from semantic or nonsemantic cues consistently produced less priming than did hearing on auditory implicit tests, extending the TAP analysis of generation to auditory priming and extending this analysis to nonsemantic generation.

\section{AUTHOR NOTE}

Correspondence concerning this article should be addressed to I. T. Z. Dew, Department of Psychology, University of North Carolina, Chapel Hill, NC 27599-3270 (e-mail: idew@unc.edu).

\section{REFERENCES}

Badgaiyan, R. D., Schacter, D. L., \& Alpert, N. M. (2001). Priming within and across modalities: Exploring the nature of rCBF increases and decreases. NeuroImage, 13, 272-282.

Bergerbest, D., Ghahremani, D. G., \& Gabrieli, J. D. E. (2004). Neural correlates of auditory repetition priming: Reduced fMRI activation in the auditory cortex. Journal of Cognitive Neuroscience, 16, 966-977.

Berry, D. C., Banbury, S., \& Henry, L. (1997). Transfer across form and modality in implicit and explicit memory. Quarterly Journal of Experimental Psychology, 50A, 1-24.

Blaxton, T. A. (1989). Investigating dissociations among memory measures: Support for a transfer-appropriate processing framework. 
Journal of Experimental Psychology: Learning, Memory, \& Cognition, 15, 657-668.

Blum, D., \& Yonelinas, A. P. (2001). Transfer across modality in perceptual implicit memory. Psychonomic Bulletin \& Review, 8, 147-154.

CANGOZ, B. (2005). Effects of anagrams with single or multiple solutions, level-of-processing, and generation on implicit memory. North American Journal of Psychology, 7, 389-405.

Carlesimo, G. A. (1999). Perceptual and conceptual components of repetition priming in anterograde amnesia. In L.-G. Nilsson \& H. J. Markowitsch (Eds.), Cognitive neuroscience of memory (pp. 213-237). Ashland, OH: Hogrefe \& Huber.

Carlesimo, G. A., Turriziani, P., \& Paulesu, E. (2004). Brain activity during intra- and cross-modal priming: New empirical data and review of the literature. Neuropsychologia, 42, 14-24.

Church, B., \& Schacter, D. L. (1994). Perceptual specificity of auditory priming: Implicit memory for voice intonation and fundamental frequency. Journal of Experimental Psychology: Learning, Memory, \& Cognition, 20, 521-533.

Clarke, R., \& Morton, J. (1983). Cross modality facilitation in tachistoscopic word recognition. Quarterly Journal of Experimental Psychology, 35A, 79-96.

Danion, J. M., Meulemans, T., Kauffmann-Muller, F., \& VerMAAT, H. (2001). Intact implicit learning in schizophrenia. American Journal of Psychiatry, 158, 944-948.

Eichenbaum, H., \& Cohen, N. J. (2001). From conditioning to conscious recollection: Memory systems of the brain. New York: Oxford University Press.

Goldwave 5.08 [Computer Software]. St. John's, NF, Canada: Goldwave.

GraF, P., \& Ryan, L. (1990). Transfer-appropriate processing for explicit and implicit memory. Journal of Experimental Psychology: Learning, Memory, \& Cognition, 16, 978-992.

Green, A. J., Easton, R., \& LaShell, L. S. R. (2001). Visual-auditory events: Cross-model perceptual priming and recognition memory. Consciousness \& Cognition, 10, 425-435.

JACOBY, L. L. (1983). Remembering the data: Analyzing interactive processes in reading. Journal of Verbal Learning \& Verbal Behavior, 22 485-508.

JELICIC, M. (1995). Aging and performance on implicit memory tasks: A brief review. International Journal of Neuroscience, 82, 155-161.

KuČERA, H., \& Francis, W. N. (1967). Computational analysis of present-day American English. Providence, RI: Brown University Press.

LeVY, B. A., \& Kirsner, K. (1989). Reprocessing text: Indirect measures of word and message level processes. Journal of Experimental Psychology: Learning, Memory, \& Cognition, 15, 407-417.

Light, L., Singh, A., \& CAPPS, J. (1986). Dissociation of memory and awareness in young and older adults. Journal of Clinical \& Experimental Neuropsychology, 8, 62-74.

Loveman, E., van Hooff, J. C., \& Gale, A. (2002). A systematic investigation of same and cross modality priming using written and spoken responses, Memory, 10, 267-276.

MacLeod, C. M., \&. Masson, M. E. J. (2000). Repetition priming in speeded word reading: Contributions of perceptual and conceptual processing episodes. Journal of Memory \& Language, 42, 208-228.

Martin-Chang, S. L., \& Levy, B. A. (2006). Word reading fluency: A transfer appropriate processing account of fluency transfer. Reading \& Writing, 19, 517-542.

Masson, M. E. J., \& MacLeod, C. M. (1992). Reenacting the route to interpretation: Enhanced perceptual identification without prior perception. Journal of Experimental Psychology: General, 121, $145-176$

Masson, M. E. J., \& MacLeod, C. M. (2002). Covert operations: Orthographic recoding as a basis for repetition priming in word identification. Journal of Experimental Psychology: Learning, Memory, \& Cognition, 28, 858-871

Mulligan, N. W. (2002). The effects of generation on conceptual implicit memory. Journal of Memory \& Language, 47, 327-342.

Mulligan, N. W. (2003). Memory: Implicit versus explicit. In L. Nadel (Ed.), Encyclopedia of cognitive science (pp. 1114-1120). London: Nature Publishing Group/MacMillan.

Mulligan, N. W., Duke, M., \& Cooper, A. W. (2007). The effects of divided attention on auditory priming. Memory \& Cognition, 35, 1245-1254

Mulligan, N. W., \& Lozito, J. P. (2004). Self generation and memory. In B. H. Ross (Ed.), The psychology of learning and motivation: Advances in research and theory (Vol. 45, pp. 175-214). San Diego: Academic Press.

Mulligan, N. M., Lozito, J. P., \& Rosner, Z. A. (2006). Generation and context memory. Journal of Experimental Psychology: Learning, Memory, \& Cognition, 32, 836-846.

Pilotti, M., Bergman, E. T., Gallo, D. A., Sommers, M., \& RoediGER, H. L., III (2000). Direct comparison of auditory implicit memory tests. Psychonomic Bulletin \& Review, 7, 347-353.

Pilotti, M., \& Beyer, T. (2002). Perceptual and lexical components of auditory repetition priming in young and older adults. Memory \& Cognition, 30, 226-236.

Pilotti, M., Gallo, D. A., \& Roediger, H. L., III (2000). Effects of hearing words, imaging hearing words, and reading on auditory implicit and explicit memory tests. Memory \& Cognition, 28, 1406-1418.

Pilotti, M., Meade, M. L., \& Gallo, D. A. (2003). Implicit and explicit measures of memory for perceptual information in young adults, healthy older adults, and patients with Alzheimer's disease. Experimental Aging Research, 29, 15-32.

RoEDIGER, H. L., III (1990). Implicit memory: Retention without remembering. American Psychologist, 45, 1043-1056.

Roediger, H. L., III, \& McDermotT, K. B. (1993). Implicit memory in normal human subjects. In F. Boller \& J. Grafman (Eds.), Handbook of neuropsychology (Vol. 8, pp. 63-131). Amsterdam: Elsevier.

SAmuelsson, S., Bogges, T. R., \& Karlsson, T. (2000). Visual implicit memory deficit and developmental surface dyslexia: A case of early occipital damage. Cortex, 36, 365-376.

SCHACTER, D. L. (1987). Implicit memory: History and current status. Journal of Experimental Psychology: Learning, Memory, \& Cognition, 13, 501-518.

SCHACTER, D. L. (1994). Priming and multiple memory systems: Perceptual mechanisms of implicit memory. In D. L. Schacter \& E. Tulving (Eds.), Memory systems 1994 (pp. 233-268). Cambridge, MA: MIT Press.

Schacter, D. L., \& Church, B. A. (1992). Auditory priming: Implicit and explicit memory for words and voices. Journal of Experimental Psychology: Learning, Memory, \& Cognition, 18, 915-930.

Schacter, D. L., Church, B. [A.], \& Treadwell, J. (1994). Implicit memory in amnesic patients: Evidence for spared auditory priming. Psychological Science, 5, 20-25.

Schacter, D. L., Dobbins, I. G., \& Schnyer, D. M. (2004). Specificity of priming: A cognitive neuroscience perspective. Nature Reviews Neuroscience, 5, 853-862.

Schacter, D. L., Wagner, A. D., \& Buckner, R. L. (2000). Memory systems of 1999. In E. Tulving \& F. I. M. Craik (Eds.), The Oxford handbook of memory (pp. 627-643). New York: Oxford University Press.

SchWARTZ, B. (1989). Effects of generation on indirect measures of memory. Journal of Experimental Psychology: Learning, Memory, \& Cognition, 15, 1119-1128.

Slamecka, N. J., \& Graf, P. (1978). The generation effect: Delineation of a phenomenon. Journal of Experimental Psychology: Human Learning \& Memory, 4, 592-604.

Smith, E. R., \& Branscombe, N. R. (1988). Category accessibility as implicit memory. Journal of Experimental Social Psychology, 24, 490-504.

Sommers, M. S. (1999). Perceptual specificity and implicit auditory priming in older and younger adults. Journal of Experimental Psychology: Learning, Memory, \& Cognition, 25, 1236-1255.

SRINIVAS, K., \& Roediger, H. L., III (1990). Classifying implicit memory tests: Category association and anagram solution. Journal of Memory \& Language, 29, 389-412.

StUART, G. P., \& JoNES, D. M. (1996). From auditory image to auditory percept: Facilitation through common processes? Memory \& Cognition, 24, 296-304.

Swick, D., Miller, K. M., \& Larsen, J. (2004). Auditory repetition priming is impaired in pure alexic patients. Brain \& Language, 89 , 543-553. 
ThorndiKe, E. L., \& LoRge, I. (1944). The teacher's word book of 30,000 words. New York: Columbia University, Teachers College.

Toth, J. P., ReINGold, E. M., \& JACOBY, L. L. (1994). Toward a redefinition of implicit memory: Process dissociations following elaborative processing and self-generation. Journal of Experimental Psychology: Learning, Memory, \& Cognition, 20, 290-303.

Verfaellie, M., Keane, M. M., \& Johnson, G. (2000). Preserved priming in auditory perceptual identification in Alzheimer's disease. Neuropsychologia, 38, 1581-1592.

WeLDON, M. S. (1991). Mechanisms underlying priming on perceptual tests. Journal of Experimental Psychology: Learning, Memory, \& Cognition, 17, 526-541.

WINNICK, W. A., \& DANIEL, S. A. (1970). Two kinds of response priming in tachistoscopic word recognition. Journal of Experimental Psychology, 84, 74-81.

\section{NOTES}

1. Research on the neural substrates of visual and auditory priming provides conflicting evidence on their separability. Some research indicates separate brain regions for visual and auditory priming (e.g., Berg- erbest, Ghahremani, \& Gabrieli, 2004; Carlesimo et al., 2004; Samuelsson, Bogges, \& Karlsson, 2000), and other studies implicate common brain areas (e.g., Badgaiyan, Schacter, \& Alpert, 2001; Swick, Miller, \& Larsen, 2004).

2. As implemented in the present study, the generation condition provided some opportunity for auditory perception of the word because the critical word was said aloud by the subject (as it was in the hear conditions). It should be noted, however, that this perceptual feedback, common to both the hear and the generation conditions, differed from the initial presentation of the stimulus in the hear conditions because it was muffled by the headphones and was presented in the subject's own voice, which, of course, did not match the voice of the words in the hear condition. Also, on the basis of the results of Experiments 1 and 2, this type of self-presentation alone does not appear to have produced significant priming. In terms of the perceptual experience with the word in the same voice and presentation form, the hear condition clearly provided a greater match between study and test than did the generation condition. We will return to this issue in the General Discussion section.

(Manuscript received April 6, 2007;

revision accepted for publication March 28, 2008.) 